\title{
Intelligent Learning Rules for Fuzzy Control of a Vibrating Screen
}

\author{
Claudio Ponce and Ernesto Ponce \\ Electronic Department, Mechanical Department, Tarapaca University, \\ Postal Code 6-D, Arica, Chile \\ eponce@uta.cl
}

\begin{abstract}
This work shows a system of intelligent control for a vibrating screen. The design is based in fuzzy logic. The set of rules was obtained by the method of group by means of the neighbor closest. The vibration of the screen (frequency and amplitude), the flow of material and the size of stones are the entrance variables. The variable of exit is the motor speed who controls the excitation force.
\end{abstract}

\section{Introduction}

In the Chilean economy the cooper is very important, CODELCO, the principal mining company has many vibratory screens. They presents several problems of efficiency and maintenance. This generates great losses due to the constant failures that take place. In order to give solution to this problem, a scale prototype of vibratory screen of last generation, was designed, [1. The vibratory screen consists of a machine with two trays with variable slope for the selection of material. It is excited by a motor and two exciting boxes. The movement of the structure is cushioned by a set of springs. The sensors measures the vibration of the structure and the level of material that arrives. An adjustable frequency drive operates on the motor, by means of a strap moves the exciting boxes.

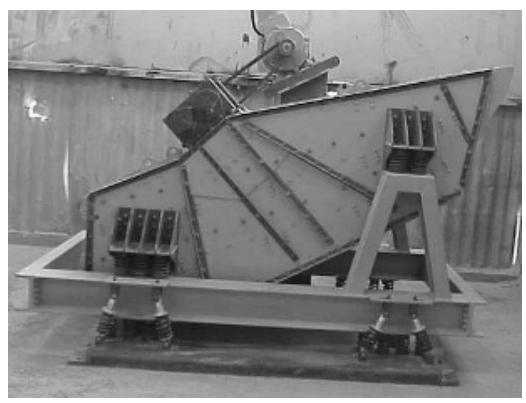

Fig. 1. Prototype on scale of a vibrating screen, used to test the intelligent control system 


\section{Structure of the Control System}

The control is made by a lodged software in a microcomputer BASIC STAMP $2 \mathrm{E}$. Software is operated with fuzzy logic. It requires of few iterations and few resources of memory. This allows a control in real time in a platform of software of low weight lodged in a very simple hardware [7].

The problem is to have a set of rules of the system. It was solved with software of intelligent learning. This one is lodged in the same microcomputer that makes the control fuzzy. Observing the variables of entrance and exit the set of control rules was deduced. The scheme of control consists of three parts:

1. A Software who takes the control of the system (Controller). This one consists of three basic stages: (a) An interface that turns the numerical variables in fuzzy variable. (b) A masterful system of control that handles the variables of entrance and exit. This operation is consulting the set of control rules. (c) An interface that turns the fuzzy variables to numerical variables.

2. A software that learns and models the system of vibratory screen (network of learning). The learning is plotted on a map of knowledge or set of rules. This knowledge is codified in machine language with instructions IF-THENELSE-WHILE. The codified information keeps in RAM (EEPROM) of the microcomputer.

3. A Software of basic control (Trainer). It gives the instructions to the controller while the learning network is training. Once the network finishes learning, the Trainer becomes disconnected. When it happens, the controller consults the new rules learned by the network of Learning.

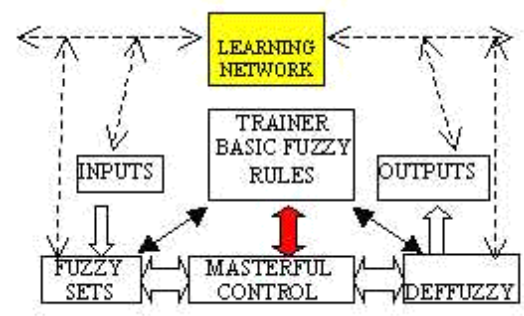

a. During the training

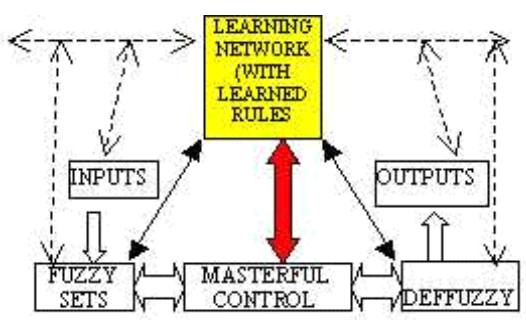

b. After the training

Fig. 2. Scheme of Control training of the intelligent controller

In the first time the controller is into operation, the program TRAINER takes the control of the MASTERFUL CONTROLLER, and the LEARNING NETWORK [4, 6], 8] will only observe the variables of entrance and exit in numerical and fuzzy form (Figure 2,a). When the LEARNING NETWORK concludes 
its training, the TRAINER will be disconnected and the LEARNING NETWORK will take the control of the MASTERFUL SYSTEM CONTROLLER (Figure 2,b).

The variables of entrance and exit are:

a. Vibration: The screen normally vibrates around $880 \mathrm{rpm}$ with a displacement of $3 \mathrm{~mm}$ and an acceleration of $1.05 \mathrm{G}$. The Amplitude and frequency are measured by means an accelerometer Memsic 2125. This accelerometer measures in two axes the impact of the vibration (amplitude and g) and rotation. The frequency can be calculated indirectly counting the maximums of the amplitude that happens in a second. This sensor is positioned in the flat $\mathrm{x}-\mathrm{y}$ of the vibration of the screen.

b. Flow Level: it is measured by means an ultrasonic sonar sensor SFR04. It works emitting a trigger of ultrasonic pulses and detecting the echo. This sensor detects the distance to the material that circulates in the belt.

c. Motor Speed: it is a three phase induction machine, with $2 \mathrm{Hp}$ of power. The speed can be controlled, adjusting the source frequency.

The motor is controlled by means an adjustable frequency drive, POWERFLEX40. The microcomputer sends an analogical signal to the drive. This signal varies between 0 volts $(0 \mathrm{~Hz})$ and 10 volts $(50 \mathrm{~Hz})$.

\section{Hardware}

The software system is lodged in a microcomputer BASICSTAMP2E, mounted in a stamp card. Its doors of exit are reinforced to give 24 volts and 1 ampere. The BASIC Stamp II is a small computer that executes programs in language PBASIC. It has 16 pins of (entered/exit) I/O that can be connected directly to digital devices or of logical levels, [2, [5].

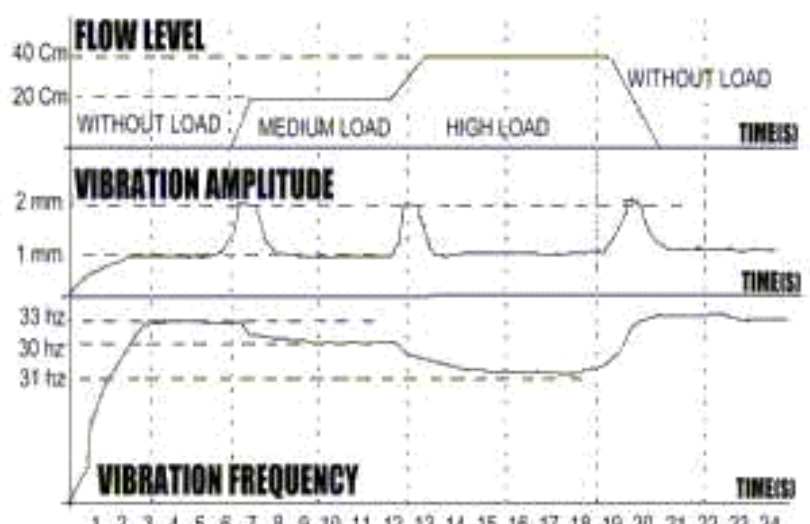

Fig. 3. Screen without load, with medium load and with high load 


\section{Experimental Results and Conclusions}

The intelligent controller was proved with satisfactory results. The Training lasted 18 hours chronological distributed in 4 days, to a programmed percentage error of a $0.5 \%$. The balance point varies between 900 and $1100 \mathrm{rpm}$ depending on the load that is applied to him. In the figure 3, it shows the screen without load, medium load and high load. In each variation of load, the vibration amplitude rises to $2 \mathrm{~mm}$ and return to $1 \mathrm{~mm}$ when the intelligent system is auto regulated.

The intelligent control is very important to optimize the operation and enlarge the life of these machines. Use a control reduces high cost in maintenance by easily avoidable failures. Thousand dollars is the loss per hour for a stopped vibrating screen and the cost of a intelligent control is around nine hundred dollars.

\section{References}

1. Ponce, E., Valdes, C., Cortes R., Development of a Vibrating Screen, FACING, Vol. 11, No. 2, pp. 35-40, 2003.

2. Nemsic Instruments, www.parallax.com

3. Narendra, K.S. and Mukhopadhyay, S., Adaptive Control Using Neural Networks and Approximate Models, IEEE Transactions on Neural Networks, Vol. 8, pp. 475485, 1997.

4. Soloway, D. and Haley, P.J., Neural Generalized Predictive Control, Proceedings of the 1996 IEEE International Symposium on Intelligent Control, pp. 277-281, 1996.

5. Basic Stamp Handbook, Parallax, www.rambal.cl

6. Hilera J. M., Redes Neuronales Artificiales, 2nd. Edition, Ed.Ra-Ma, Madrid, Spain, 2002.

7. D.Dubois and H.Prade, Fuzzy sets and systems, 3rd Edition, Ed.Public Press, USA, 2003.

8. Kosko, B., Neural Networks and Fuzzy Systems, 4th Edition, Ed.Prentice-Hall, 2003. 